Bull. Austral. Math. Soc.

VOL. $71(2005)$ [205-213]

\title{
INVERSE SEMIGROUPS GENERATED BY LINEAR TRANSFORMATIONS
}

\author{
Suzana Mendes-Gonçalves and R.P. Sullivan
}

\begin{abstract}
Suppose $X$ is a set with $|X|=p \geqslant q \geqslant \aleph_{0}$ and let $B=B L(p, q)$ denote the Baer-Levi semigroup defined on $X$. In 1984, Howie and Marques-Smith showed that, if $p=q$, then $B B^{-1}=I(X)$, the symmetric inverse semigroup on $X$, and they described the subsemigroup of $I(X)$ generated by $B^{-1} B$. In 1994, Lima extended that work to 'independence algebras', and thus also to vector spaces. In this paper, we answer the natural question: what happens when $p>q$ ? We also show that, in this case, the analogues $B B^{-1}$ for sets and $G G^{-1}$ for vector spaces are never isomorphic, despite their apparent similarities.
\end{abstract}

\section{INTRODUCTION}

Let $X$ be an infinite set with cardinal $p$, and let $q$ be a cardinal such that $\aleph_{0} \leqslant q \leqslant p$. Let $P(X)$ denote the set of all partial transformations of $X$ : that is, all transformations $\alpha$ whose domain, dom $\alpha$, and range, $\operatorname{ran} \alpha$, are subsets of $X$. As usual, the composition $\alpha \circ \beta$ of $\alpha, \beta \in P(X)$ is the transformation with domain $Y=(\operatorname{ran} \alpha \cap \operatorname{dom} \beta) \alpha^{-1}$ such that, for all $x \in Y$,

$$
x(\alpha \circ \beta)=(x \alpha) \beta,
$$

and we often write $\alpha \circ \beta$ more simply as $\alpha \beta$ (compare [1, vol 1, p. 29]). Obviously, $Y$ is a subset of $X$ and $\alpha \circ \beta \in P(X)$ if $\alpha, \beta \in P(X)$. Indeed, it is well-known that $(P(X), \circ)$ is a semigroup. Let $T(X)$ denote the subsemigroup of $P(X)$ consisting of all $\alpha \in P(X)$ with domain $X$, and let $I(X)$ denote the symmetric inverse semigroup on $X$ : that is, the set of all injective elements of $P(X)$.

If $\alpha \in P(X)$, we let

$$
\begin{array}{ll}
c(\alpha)=\left|\cup\left\{y \alpha^{-1}:\left|y \alpha^{-1}\right| \geqslant 2\right\}\right|, & r(\alpha)=|\operatorname{ran} \alpha| \\
g(\alpha)=|X \backslash \operatorname{dom} \alpha|, & d(\alpha)=|X \backslash \operatorname{ran} \alpha|
\end{array}
$$

Received 4th October, 2004

This paper forms part of work by the first author for a PhD supervised by the second author; both authors gratefully acknowledge the support of the Portuguese Foundation for Science and Technology (FCT) through the research program POCTI.

Copyright Clearance Centre, Inc. Serial-fee code: 0004-9727/05 \$A2.00+0.00. 
and refer to these cardinal numbers as the collapse, rank, gap and defect of $\alpha$, respectively. We now write

$$
B=B L(p, q)=\{\alpha \in T(X): c(\alpha)=0, d(\alpha)=q\}
$$

which is the Baer-Levi semigroup on $X$ of type $(p, q)$ as discussed in [1, section 8.1]. In [2, Theorems 2.3 and 2.4], the authors showed that if $p=q$, then

$$
\begin{aligned}
B^{-1} & =\{\alpha \in I(X): g(\alpha)=p, d(\alpha)=0\} \\
B B^{-1} & =I(X) \text { and } B^{-1} B=\{\alpha \in I(X): r(\alpha)=g(\alpha)=d(\alpha)=p\}
\end{aligned}
$$

In this case (namely, $p=q$ ), they also showed that the subsemigroup of $I(X)$ generated by $B^{-1} B$ equals $K_{p}=\{\alpha \in I(X): g(\alpha)=d(\alpha)=p\}$ ([2, Theorem 2.5]). In [2, Theorem $3.2]$, the authors proved that $K_{p}$ is, in fact, the inverse subsemigroup of $I(X)$ generated by the nilpotent elements of index 2 (that is, all $\alpha \in I(X)$ for which $\alpha \neq \emptyset$ and $\alpha^{2}=\emptyset$ ). In [3, Proposition 4.3, Proposition 4.5 and Theorem 5.6], Lima extended these results to independence algebras, and thus also to vector spaces ('sets' and 'vector spaces' are prime examples of an independence algebra). Here, we answer the natural question: what happens when $p>q$ ?

\section{MAIN RESUlTS}

Let $V$ be a vector space over a field $F$ and suppose $\operatorname{dim} V=p \geqslant \aleph_{0}$. We let $P(V)$ denote the set of all partial linear transformations of $V$ : that is, all linear transformations $\alpha: A \rightarrow B$ where $A, B$ are subspaces of $V$. As for partial transformations of a set, we denote the domain and the range of $\alpha \in P(V)$ by dom $\alpha$ and $\operatorname{ran} \alpha$, respectively, and we define the composition $\alpha \circ \beta$ of $\alpha, \beta \in P(V)$ to be the linear transformation with domain $U=(\operatorname{ran} \alpha \cap \operatorname{dom} \beta) \alpha^{-1}$ such that, for all $u \in U$,

$$
u(\alpha \circ \beta)=(u \alpha) \beta .
$$

To simplify notation, we often write $\alpha \circ \beta$ as $\alpha \beta$. Clearly, $U$ is a subspace of $V$ and $\alpha \circ \beta \in P(V)$ if $\alpha, \beta \in P(V)$. Also, $(\alpha \circ \beta) \circ \gamma=\alpha \circ(\beta \circ \gamma)$ for all $\alpha, \beta, \gamma \in P(V)$, so $(P(V), \circ)$ is a semigroup. Let $T(V)$ denote the subsemigroup of $P(V)$ consisting of all linear transformations with domain $V$, and let $I(V)$ denote the set of all injective partial linear transformations of $V$. It is easy to see that $I(V)$ is an inverse subsemigroup of $P(V)$ and its idempotents are precisely the identity maps id $U$ on the subspaces $U$ of $V$. Note that we use the ' $V$ ' in place of ' $X$ ' to denote the fact that now we are considering linear transformations.

As an abbreviation, we write $\left\{e_{i}\right\}$ to denote a subset $\left\{e_{i}: i \in I\right\}$ of $V$, taking as understood that the subscript $i$ belongs to some (unmentioned) index set $I$. The subspace $A$ of $V$ generated by a linearly independent subset $\left\{e_{i}\right\}$ of $V$ is denoted by $\left\langle e_{i}\right\rangle$, and we write $\operatorname{dim} A=|I|$. Often it is necessary to define some $\alpha \in P(V)$ by first choosing a 
linearly independent subset $\left\{e_{i}\right\}$ of $V$ and some $\left\{a_{i}\right\} \subseteq V$, and then letting $e_{i} \alpha=a_{i}$ for each $i$ and extending this action by linearity to the whole of dom $\alpha=\left\langle e_{i}\right\rangle$. To abbreviate matters, we simply say, given $\left\{e_{i}\right\}$ and $\left\{a_{i}\right\}$ within context, that $\alpha \in P(V)$ is defined by letting

$$
\alpha=\left(\begin{array}{c}
e_{i} \\
a_{i}
\end{array}\right) .
$$

Similar notation for $P(X)$ is now standard: for example, see [4].

If $\alpha \in P(V)$, we write $\operatorname{ker} \alpha$ for the kernel of $\alpha$, and put

$$
\begin{array}{lll}
n(\alpha)=\operatorname{dim} \operatorname{ker} \alpha, & r(\alpha)=\operatorname{dim} \operatorname{ran} \alpha, \\
g(\alpha)=\operatorname{codim} \operatorname{dom} \alpha, & d(\alpha)=\operatorname{codim} \operatorname{ran} \alpha .
\end{array}
$$

As usual, these are called the nullity, rank, gap and defect of $\alpha$, respectively. For each cardinal $q$ such that $\aleph_{0} \leqslant q \leqslant p$, consider the linear Baer-Levi semigroup on $V$ :

$$
G=G S(p, q)=\{\alpha \in T(V): n(\alpha)=0, d(\alpha)=q\} .
$$

As shown in [5], this is a right simple, right cancellative subsemigroup of $T(V)$ without idempotents. Thus, it is not an inverse semigroup: in fact, $\alpha \in G$ if and only if $\alpha^{-1} \in G^{-1}$ where

$$
G^{-1}=\{\beta \in I(V): g(\beta)=q, d(\beta)=0\} .
$$

Moreover, from the anti-isomorphism $G \rightarrow G^{-1}, \alpha \mapsto \alpha^{-1}$, we see that $G^{-1}$ is a left simple, left cancellative subsemigroup of $I(V)$ which has no idempotents. As already remarked, Lima [3, Propositions 4.3 and 4.5], showed that if $p=q$, then

$$
G G^{-1}=I(V) \text { and } G^{-1} G=\{\alpha \in I(V): r(\alpha)=g(\alpha)=d(\alpha)=p\} .
$$

Therefore, in this case, $G G^{-1}$ is an inverse semigroup but $G^{-1} G$ is not even closed. We shall show that if $p>q$, then both $G G^{-1}$ and $G^{-1} G$ are inverse subsemigroups of $I(V)$.

Henceforth, we let 0 denote the linear map with domain $\{0\}$ in $V$ : note that this mapping is a zero for the semigroup $I(V)$.

LEMmA 1. Suppose $\aleph_{0} \leqslant q<p$ and let $G, G^{-1}$ be as defined in (1) and (2). If $\alpha \in G$ and $\beta \in G^{-1}$ then $\operatorname{dim}(\operatorname{ran} \alpha \cap \operatorname{dom} \beta)=p$. In particular, $\alpha \beta \neq 0$ in $I(V)$.

Proof: Suppose $\left\{e_{i}\right\}$ is a basis for $\operatorname{ran} \alpha \cap \operatorname{dom} \beta$ and $|I|<p$. Since $q<p$, we know

$$
\operatorname{dim}(\operatorname{ran} \alpha)=p=\operatorname{dim}(\operatorname{dom} \beta),
$$

so $\left\{e_{i}\right\}$ can be expanded to bases $\left\{e_{i}\right\} \cup \dot{\cup}\left\{a_{j}\right\}$ for $\operatorname{ran} \alpha$ and $\left\{e_{i}\right\} \dot{\cup}\left\{b_{j}\right\}$ for dom $\beta$ where $|J|=p$. Then $\left\{e_{i}\right\} \dot{\cup}\left\{a_{j}\right\} \dot{\cup}\left\{b_{j}\right\}$ is linearly independent: for, if

$$
\sum x_{i} e_{i}+\sum y_{j} a_{j}+\sum z_{j} b_{j}=0
$$


for some scalars $x_{i}, y_{j}$ and $z_{j}$, then

$$
\sum z_{j} b_{j} \in \operatorname{ran} \alpha \cap \operatorname{dom} \beta,
$$

and so $\sum z_{j} b_{j}=\sum r_{i} e_{i}$ for some scalars $r_{i}$. Thus,

$$
\sum r_{i} e_{i}-\sum z_{j} b_{j}=0
$$

and so $z_{j}=0$ for each $j$. Therefore, $\sum x_{i} e_{i}+\sum y_{j} a_{j}=0$ and, since the set $\left\{e_{i}\right\} \dot{\cup}\left\{a_{j}\right\}$ is linearly independent, it follows that $x_{i}=0$ for each $i$ and $y_{j}=0$ for each $j$. Consequently, $d(\alpha) \geqslant|J|=p$, a contradiction.

Recall that $\operatorname{dom}(\alpha \beta) \subseteq \operatorname{dom} \alpha$ and $\operatorname{ran}(\alpha \beta) \subseteq \operatorname{ran} \beta$, so $g(\alpha \beta) \geqslant g(\alpha)$ and $d(\alpha \beta)$ $\geqslant d(\beta)$. Hence the next result is a little surprising.

TheOREM 1. Suppose $\aleph_{0} \leqslant q<p$. If $G$ and $G^{-1}$ are as defined in (1) and (2), then

$$
G G^{-1}=\{\alpha \in I(V): g(\alpha) \leqslant q, d(\alpha) \leqslant q\}
$$

and this is an inverse subsemigroup of $I(V)$ without nilpotents.

ProOf: Let $\alpha \in G$ and $\beta \in G^{-1}$. Then, from Lemma $1, \operatorname{dim}(\operatorname{ran} \alpha \cap \operatorname{dom} \beta)=p$. Suppose $\left\{e_{i}\right\}$ is a basis for $\operatorname{ran} \alpha$ ndom $\beta$ and expand it to bases $\left\{e_{i}\right\} \dot{\cup}\left\{a_{r}\right\}$ and $\left\{e_{i}\right\} \dot{\cup}\left\{b_{s}\right\}$ for $\operatorname{ran} \alpha$ and $\operatorname{dom} \beta$, respectively. Since $\alpha$ is one-to-one, there exist unique $f_{i}$ and $f_{r}$ such that $e_{i} \alpha^{-1}=f_{i}$ and $a_{r} \alpha^{-1}=f_{r}$. Write $e_{i} \beta=g_{i}$ for each $i$ and $b_{s} \beta=g_{s}$ for each $s$. We have $V=\left\langle f_{i}, f_{r}\right\rangle=\left\langle g_{i}, g_{s}\right\rangle$ and

$$
\alpha=\left(\begin{array}{ll}
f_{i} & f_{r} \\
e_{i} & a_{r}
\end{array}\right), \beta=\left(\begin{array}{ll}
e_{i} & b_{s} \\
g_{i} & g_{s}
\end{array}\right)
$$

Therefore

$$
\alpha \beta=\left(\begin{array}{c}
f_{i} \\
g_{i}
\end{array}\right)
$$

and so $g(\alpha \beta)=\operatorname{dim}\left\langle f_{r}\right\rangle=|R|$ and $d(\alpha \beta)=\operatorname{dim}\left\langle g_{s}\right\rangle=|S|$. As in the proof of Lemma 1, $\left\{e_{i}\right\} \dot{\cup}\left\{a_{r}\right\} \dot{\cup}\left\{b_{s}\right\}$ is linearly independent, so it can be expanded to a basis for $V$, say

$$
\left\{e_{i}\right\} \dot{\cup}\left\{a_{r}\right\} \dot{\cup}\left\{b_{s}\right\} \dot{\cup}\left\{c_{\ell}\right\} \text {. }
$$

Thus,

$$
|R| \leqslant|R|+|L|=g(\beta)=q
$$

and

$$
|S| \leqslant|S|+|L|=d(\alpha)=q .
$$

Hence, $\alpha \beta$ is such that $g(\alpha \beta) \leqslant q$ and $d(\alpha \beta) \leqslant q$.

Conversely, let $\alpha: A \rightarrow B$ be an injective linear map such that $g(\alpha) \leqslant q$ and $d(\alpha) \leqslant q$. Suppose $\left\{a_{i}\right\}$ is a basis for $A$ and write $a_{i} \alpha=b_{i}$ for each $i$. Then $\left\{b_{i}\right\}$ is a basis 
for ran $\alpha$. Expand $\left\{a_{i}\right\}$ and $\left\{b_{i}\right\}$ to bases $\left\{a_{i}\right\} \cup \dot{\cup}\left\{a_{j}\right\}$ and $\left\{b_{i}\right\} \cup \dot{\cup}\left\{b_{\ell}\right\}$ for $V$, respectively, with $|J|=g(\alpha) \leqslant q,|L|=d(\alpha) \leqslant q$. Since $|L| \leqslant q<p$, we may write $\left\{a_{i}\right\}$ as $\left\{u_{i}\right\} \dot{\cup}\left\{u_{k}\right\}$ with $|K|=q$ and $\left\{u_{k}\right\}=\left\{v_{k}\right\} \cup \dot{\cup}\left\{v_{\ell}\right\}$. Now define in $P(V)$

$$
\beta=\left(\begin{array}{ll}
a_{i} & a_{j} \\
u_{i} & a_{j}
\end{array}\right), \quad \gamma=\left(\begin{array}{ll}
u_{i} & v_{\ell} \\
b_{i} & b_{\ell}
\end{array}\right) .
$$

It is easy to see that $\beta, \gamma \in I(V)$ and $g(\beta)=0, d(\beta)=\operatorname{dim}\left\langle u_{k}\right\rangle=q, d(\gamma)=0$ and $g(\gamma)=\operatorname{dim}\left\langle v_{k}, a_{j}\right\rangle=q$. Therefore, $\beta \in G$ and $\gamma \in G^{-1}$. Since $\alpha=\beta \gamma$, it follows that $\alpha \in G G^{-1}$.

Next, we show that $G G^{-1}$ is an inverse semigroup. To do so, let $\alpha, \beta \in G G^{-1}$. Then, $g(\alpha) \leqslant q, d(\alpha) \leqslant q, g(\beta) \leqslant q$ and $d(\beta) \leqslant q$. Since $q<p, \operatorname{dim}(\operatorname{dom} \alpha)=p=\operatorname{dim}(\operatorname{dom} \beta)$. Suppose $\left\{a_{i}\right\}$ is a basis for dom $\alpha$ and $\left\{b_{i}\right\}$ a basis for dom $\beta$. Write $a_{i} \alpha=u_{i}$ and $b_{i} \beta=v_{i}$ for each $i$. Suppose ran $\alpha \cap \operatorname{dom} \beta=\{0\}$. Then, $\left\{u_{i}\right\} \cup \dot{\cup}\left\{b_{i}\right\}$ is linearly independent and it can be expanded to a basis $\left\{u_{i}\right\} \dot{\cup}\left\{b_{i}\right\} \dot{\cup}\left\{c_{\ell}\right\}$ for $V$. Thus, $q \geqslant d(\alpha)=\operatorname{dim}\left(b_{i}, c_{\ell}\right\rangle$ $=|I|+|L|=p$, a contradiction. Therefore, $\operatorname{ran} \alpha \cap \operatorname{dom} \beta \neq\{0\}$. Let $\left\{e_{j}\right\}$ be a basis for $\operatorname{ran} \alpha \cap \operatorname{dom} \beta$ and expand it to bases $\left\{e_{j}\right\} \dot{\cup}\left\{u_{r}\right\}$ and $\left\{e_{j}\right\} \dot{\cup}\left\{b_{s}\right\}$ for $\operatorname{ran} \alpha$ and $\operatorname{dom} \beta$, respectively. Since $\alpha$ is one-to-one, there exist unique $f_{j}$ and $f_{r}$ such that $f_{j} \alpha=e_{j}$ and $f_{r} \alpha=u_{r}$. Write $e_{j} \beta=v_{j}$ and $b_{s} \beta=v_{s}$. Then, we have

$$
\alpha=\left(\begin{array}{ll}
f_{j} & f_{r} \\
e_{j} & u_{r}
\end{array}\right), \beta=\left(\begin{array}{ll}
e_{j} & b_{s} \\
v_{j} & v_{s}
\end{array}\right) \text { and so } \alpha \beta=\left(\begin{array}{l}
f_{j} \\
v_{j}
\end{array}\right) .
$$

Since $\left\{e_{j}\right\} \dot{\cup}\left\{u_{r}\right\} \dot{\cup}\left\{b_{s}\right\}$ is linearly independent, it can be expanded to a basis

$$
\left\{e_{j}\right\} \dot{\cup}\left\{u_{r}\right\} \dot{\cup}\left\{b_{s}\right\} \dot{\cup}\left\{d_{t}\right\}
$$

for $V$. Thus, $q \geqslant d(\alpha)=|S|+|T|$ and $q \geqslant g(\beta)=|R|+|T|$ and so $|S|,|R| \leqslant q$. Hence, $g(\alpha \beta)=|R|+g(\alpha) \leqslant q$ and $d(\alpha \beta)=|S|+d(\beta) \leqslant q$. Therefore, $\alpha \beta \in G G^{-1}$ and this shows that $G G^{-1}$ is a semigroup. Since $\operatorname{dom} \alpha^{-1}=\operatorname{ran} \alpha$ and $\operatorname{ran} \alpha^{-1}=\operatorname{dom} \alpha$, it follows that $g\left(\alpha^{-1}\right)=d(\alpha)$ and $d\left(\alpha^{-1}\right)=g(\alpha)$ and hence $G G^{-1}$ is an inverse semigroup. Moreover, since $d(\alpha \beta) \leqslant q<p$ for each $\alpha, \beta \in G G^{-1}$, we deduce that $r(\alpha \beta)=p$ and thus $\alpha \beta \neq 0$ : that is, $G G^{-1}$ has no nilpotents.

REMARK. For the above proof, it is natural to think $G G^{-1} G \subseteq G$, but this does not hold. To see this, suppose $\left\{e_{i}\right\}$ is a basis for $V$ and write $\left\{e_{i}\right\}$ as $\left\{f_{i}\right\} \dot{\cup}\left\{f_{j}\right\}$ and $\left\{f_{j}\right\}$ as $\left\{a_{j}\right\} \cup \dot{\cup}\left\{b_{j}\right\}$ where $|J|=q$. Now define $\alpha, \beta \in I(V)$ by

$$
\alpha=\left(\begin{array}{ll}
f_{i} & f_{j} \\
f_{i} & a_{j}
\end{array}\right), \beta=\left(\begin{array}{ll}
f_{i} & b_{j} \\
f_{i} & f_{j}
\end{array}\right) .
$$

Clearly, $\operatorname{dom} \alpha=V=\operatorname{ran} \beta, d(\alpha)=\operatorname{dim}\left\langle b_{j}\right\rangle=q$ and $g(\beta)=\operatorname{dim}\left\langle a_{j}\right\rangle=q$. Thus, $\alpha \in G$ and $\beta \in G^{-1}$. Since $\operatorname{dom}(\alpha \beta \gamma) \subseteq \operatorname{dom}(\alpha \beta)=\left\langle f_{i}\right\rangle \neq V$ for every $\gamma \in G$, it follows that $\alpha \beta \gamma \notin G$. 
THEOREM 2. Suppose $\aleph_{0} \leqslant q<p$. If $G$ and $G^{-1}$ are as defined in (1) and (2), then

$$
G^{-1} G=\{\alpha \in I(V): g(\alpha)=d(\alpha)=q\}
$$

and this is an inverse subsemigroup of $I(V)$ without nilpotents.

Proof: Let $\alpha \in G$ and $\beta \in G^{-1}$. Then, $\operatorname{dom} \alpha=V=\operatorname{ran} \beta$ and so $\operatorname{dom}(\beta \alpha)$ $=(\operatorname{ran} \beta \cap \operatorname{dom} \alpha) \beta^{-1}=V \beta^{-1}=\operatorname{dom} \beta$ and $\operatorname{ran}(\beta \alpha)=V \alpha=\operatorname{ran} \alpha$. Therefore, $g(\beta \alpha)$ $=g(\beta)=q$ and $d(\beta \alpha)=d(\alpha)=q$.

Conversely, let $\alpha \in I(V)$ be such that $g(\alpha)=d(\alpha)=q$ and suppose $\left\{a_{i}\right\}$ is a basis for $\operatorname{dom} \alpha$. Write $a_{i} \alpha=b_{i}$ for each $i$ and expand $\left\{a_{i}\right\}$ and $\left\{b_{i}\right\}$ to bases for $V$, say $\left\{a_{i}\right\} \dot{\cup}\left\{a_{j}\right\}$ and $\left\{b_{i}\right\} \dot{\cup}\left\{b_{j}\right\}$, respectively, with $|J|=q$. Since $q<p=\operatorname{dim} V$, it follows that $|I|=p$. Suppose $\left\{e_{i}\right\}$ is a basis for $V$ and define in $I(V)$

$$
\beta=\left(\begin{array}{l}
a_{i} \\
e_{i}
\end{array}\right), \gamma=\left(\begin{array}{l}
e_{i} \\
b_{i}
\end{array}\right)
$$

Then, $g(\beta)=\operatorname{dim}\left\langle a_{j}\right\rangle=g(\alpha)=q, \operatorname{ran} \beta=V=\operatorname{dom} \gamma$ and $d(\gamma)=\operatorname{dim}\left\langle b_{j}\right\rangle=d(\alpha)=q$. Therefore, $\beta \in G^{-1}$ and $\gamma \in G$. Since $\alpha=\beta \gamma$, we have $\alpha \in G^{-1} G$ and the result follows.

To see that $G^{-1} G$ is a semigroup, let $\alpha, \beta \in G^{-1} G$. Then, $g(\alpha)=d(\alpha)=g(\beta)$ $=d(\beta)=q$ and, since $q<p, \operatorname{dim}(\operatorname{dom} \alpha)=p=\operatorname{dim}(\operatorname{dom} \beta)$. Let $\left\{a_{i}\right\}$ and $\left\{b_{i}\right\}$ be bases for $\operatorname{dom} \alpha$ and $\operatorname{dom} \beta$, respectively, and write $a_{i} \alpha=e_{i}$ and $b_{i} \beta=f_{i}$ for each $i$. Suppose $\operatorname{ran} \alpha \cap \operatorname{dom} \beta=\{0\}$. Then $\left\{e_{i}\right\} \dot{\cup}\left\{b_{i}\right\}$ is linearly independent and so it can be expanded to a basis for $V$, say $\left\{e_{i}\right\} \dot{\cup}\left\{b_{i}\right\} \dot{\cup}\left\{v_{j}\right\}$. Thus, $q=d(\alpha)=\operatorname{dim}\left\langle b_{i}, v_{j}\right\rangle=p$, which contradicts our assumption on $q$ and $p$. Therefore, $\operatorname{ran} \alpha \cap \operatorname{dom} \beta \neq\{0\}$. Let $\left\{c_{\ell}\right\}$ be a basis for $\operatorname{ran} \alpha \cap \operatorname{dom} \beta$ and expand it to bases $\left\{c_{\ell}\right\} \dot{\cup}\left\{e_{r}\right\}$ and $\left\{c_{\ell}\right\} \dot{\cup}\left\{b_{s}\right\}$ for $\operatorname{ran} \alpha$ and $\operatorname{dom} \beta$, respectively. Since $\alpha$ is injective, there exist unique $u_{\ell}$ and $u_{r}$ in $V$ such that $u_{\ell} \alpha=c_{\ell}$ and $u_{r} \alpha=e_{r}$. If we write $c_{\ell} \beta=f_{\ell}$ for each $\ell$ and $b_{s} \beta=f_{s}$ for each $s$, we then have

$$
\alpha=\left(\begin{array}{ll}
u_{\ell} & u_{r} \\
c_{\ell} & e_{r}
\end{array}\right), \beta=\left(\begin{array}{ll}
c_{\ell} & b_{s} \\
f_{\ell} & f_{s}
\end{array}\right)
$$

and so

$$
\alpha \beta=\left(\begin{array}{l}
u_{\ell} \\
f_{\ell}
\end{array}\right) .
$$

As in the proof of Lemma 1, it follows that $\left\{c_{\ell}\right\} \dot{\cup}\left\{e_{r}\right\} \dot{\cup}\left\{b_{s}\right\}$ is linearly independent, so it can be expanded to a basis $\left\{c_{\ell}\right\} \dot{\cup}\left\{e_{r}\right\} \dot{\cup}\left\{b_{s}\right\} \dot{\cup}\left\{d_{k}\right\}$ for $V$. Therefore, $|S| \leqslant|S|+|K|$ $=\operatorname{codim}\left\langle c_{\ell}, e_{r}\right\rangle=d(\alpha)=q$ and $|R| \leqslant|R|+|K|=\operatorname{codim}\left\langle c_{\ell}, b_{s}\right\rangle=g(\beta)=q$. Hence, $g(\alpha \beta)=|R|+g(\alpha)=q$ and $d(\alpha \beta)=|S|+d(\beta)=q$. Thus, $\alpha \beta \in G^{-1} G$ and so $G^{-1} G$ is a semigroup. Since $g\left(\alpha^{-1}\right)=d(\alpha)$ and $d\left(\alpha^{-1}\right)=g(\alpha)$, it follows that $G G^{-1}$ is an inverse semigroup. 


\section{ISOMORPHISM PROBLEM}

Of course, there are analogues of Theorems 1 and 2 for sets. That is, if $X$ is a set and $\aleph_{0} \leqslant q<p=|X|$, we can show, in a manner similar to the above proofs, that

$$
\begin{aligned}
& B B^{-1}=\{\alpha \in I(X): g(\alpha) \leqslant q, d(\alpha) \leqslant q\} \\
& B^{-1} B=\{\alpha \in I(X): g(\alpha)=d(\alpha)=q\}
\end{aligned}
$$

and that these are inverse subsemigroups of $I(X)$ without nilpotents (indeed, the above proofs hold almost verbatim, provided we omit all references to bases and their extension). Since these semigroups look so much like $G G^{-1}$ and $G^{-1} G$, an obvious problem is to decide whether the corresponding pairs are isomorphic.

In [6] we showed that $I(X)$ and $I(V)$ are almost never isomorphic. Here we use a similar idea to show the above corresponding pairs are never isomorphic.

The idempotents of $I(X)$ have the form id where $_{Y} \subseteq X$; and they are partially ordered by

$$
\mathrm{id}_{A} \leqslant \mathrm{id}_{B} \Longleftrightarrow \operatorname{id}_{A}=\mathrm{id}_{A} \circ \mathrm{id}_{B} \Longleftrightarrow A \subseteq B \text {. }
$$

We say $\mathrm{id}_{B}$ covers $\mathrm{id}_{A}$, and write $\mathrm{id}_{A}<\mathrm{id}_{B}$, if there is no idempotent in $I(X)$ strictly between $\mathrm{id}_{A}$ and $\mathrm{id}_{B}$ under this partial order: in other words, when this occurs, $B=A \cup\{x\}$ for some $x \notin A$. In addition, if $M \subseteq X$ and there are distinct $a, b \notin M$, we say

$$
\operatorname{id}_{M}<\operatorname{id}_{M \cup\{a\}}<\operatorname{id}_{M \cup\{a, b\}}
$$

is an idempotent chain of length 2 ; and that $\alpha \in I(X)$ preserves this chain if

$$
\operatorname{id}_{M} \circ \alpha=\operatorname{id}_{M} \text { and } \operatorname{id}_{M \cup\{a, b\}}=\alpha \alpha^{-1} \text {. }
$$

Clearly, when this happens, $a \alpha=x$ and $b \alpha=y$ for some $x \neq y$, and hence

$$
\operatorname{id}_{M}<\operatorname{id}_{M \cup\{x\}}<\operatorname{id}_{M \cup\{x, y\}}
$$

where $\mathrm{id}_{M \cup\{a\}} \circ \alpha=\mathrm{id}_{M \cup\{x\}}$ and $\mathrm{id}_{M \cup\{a, b\}} \circ \alpha=\mathrm{id}_{M \cup\{x, y\}}$ (this explains the terminology).

Let $S$ denote either $B B^{-1}$ and $B^{-1} B$, and let $D_{2}(S)$ equal the set of all $\alpha \in S$ of the form:

$$
\alpha=\left(\begin{array}{ll}
a & b \\
x & y
\end{array}\right) \cup \mathrm{id}_{M},
$$

where $a \neq b, x \neq y, M \subseteq X$, and none of $a, b, x, y$ belong to $M$. Clearly, $D_{2}(S)$ is non-empty, and we assert that its elements are characterised by the statement:

$$
\alpha \in S \text { and } \alpha \text { preserves an idempotent chain in } S \text { of length } 2 \text {. }
$$


Clearly, if $\alpha \in S$ has the form in (4) then $|X \backslash M| \leqslant q$, hence both id ind $_{M}$ id M $_{M}\{a\}$ belong to $S$, and so $\alpha$ preserves the idempotent chain of length 2 in (3). Conversely, suppose the chain in (3) belongs to $S$ and assume $\alpha \in S$ preserves it. Then, by definition, dom $\alpha=M \cup\{a, b\}$ and $\operatorname{id}_{M} \subseteq \alpha$; and moreover, since $\alpha$ is injective, we have $a \alpha=x$ and $b \alpha=y$ for some $x \neq y$, and so $\alpha$ has the form in (4).

Next we assert that $S$ satisfies:

if $\alpha \in S$ preserves a chain $\operatorname{id}_{M}<\operatorname{id}_{M \cup\{a\}}<\operatorname{id}_{M \cup\{a, b\}}$ and $\alpha \gamma, \alpha^{2} \gamma \neq \mathrm{id}_{M}$ for some idempotent $\gamma \in S$ that covers $\mathrm{id}_{M}$, and if $\alpha^{2}$ is not idempotent, then $\gamma \alpha=\mathrm{id}_{M}$.

To see this, suppose $\alpha \in S$ satisfies the initial condition, hence it has the form in (4), and assume an idempotent $\gamma \in S$ covers $\mathrm{id}_{M}$ and $\alpha \gamma \neq \mathrm{id}_{M}$. Then $\operatorname{ran} \alpha \cap \operatorname{dom} \gamma \neq M$ and so, without loss of generality,

$$
\gamma=\left(\begin{array}{l}
x \\
x
\end{array}\right) \cup \operatorname{id}_{M} .
$$

Suppose $x=a$ : in this case, if $b=y$ then $\alpha=\mathrm{id}_{\{a, b\}} \cup \mathrm{id}_{M}$ is idempotent; and if $b \neq y$ then $\alpha^{2}=a_{a} \cup \mathrm{id}_{M}$ is idempotent, contradicting the supposition in both cases. On the other hand, if $x=b$ then $y \neq b$ (since $\alpha$ is injective) and $y \neq a$ (since $\alpha^{2}$ is not idempotent). Hence, in this case, $\alpha^{2}=a_{y} \cup \mathrm{id}_{M}$, so $\alpha^{2} \gamma \neq \mathrm{id}_{M}$ implies $y \in \operatorname{dom} \gamma$, which is impossible since $y \notin M \cup\{x\}$. Therefore, $x \notin\{a, b\}$ and it follows that $\gamma \alpha=\mathrm{id}_{M}$.

Clearly, property $(*)$ will be preserved under an isomorphism $\varphi$ from $B B^{-1}$ onto $G G^{-1}$ (and likewise for the other pair of analogues). This is because the notions of 'cover' and 'idempotent chain of length 2' can be described algebraically, and also because $B B^{-1}$ is an inverse semigroup (hence, both $\alpha^{-1}$ and $\alpha \alpha^{-1}$ belong to $B B^{-1}$ if $\alpha \in B B^{-1}$ ). However, we assert that the image of $S$ under $\varphi$ does not satisfy (*).

To see this, choose linearly independent $a, b \in V$, then $\{b, a+b\}$ is also linearly independent. Suppose $N$ is a subspace of $V$ such that $N \cap\langle a, b\rangle=\{0\}$ and assume

$$
\beta=\left(\begin{array}{cc}
a & b \\
b & a+b
\end{array}\right) \cup \operatorname{id}_{N} \in S \varphi
$$

(this containment simply restricts the codimension of $N$ as required). Now $\beta$ preserves the idempotent chain of length 2 in $S \varphi$ given by

$$
\operatorname{id}_{N}<\mathrm{id}_{N+\langle a\rangle}<\mathrm{id}_{N+\langle a, b\rangle}
$$

since $\operatorname{id}_{N} \circ \beta=\operatorname{id}_{N}$ and $\operatorname{id}_{N+\langle a, b\rangle}=\beta \beta^{-1}$. Hence $\beta$ is the image of some element of $D_{2}(S)$. However, $\beta$ does not satisfy $(*)$. For, clearly $\beta^{2}$ is not idempotent. Also, if $c=a+b$ then $\gamma=\mathrm{id}_{N+\langle c\rangle}$ is an idempotent in $S \varphi$ which covers $\mathrm{id}_{N}$. In addition, $\beta \gamma \neq \mathrm{id}_{N}$ and $\beta^{2} \gamma \neq \mathrm{id}_{N}$. However, also $\gamma \beta \neq \mathrm{id}_{N}$, and the last assertion follows. 
The above argument shows that, despite their similarities, these corresponding pairs of semigroups are essentially different, something which is not apparent when they are regarded as semigroups of endomorphisms of an independence algebra.

\section{REFERENCES}

[1] A.H. Clifford and G.B. Preston, The algebraic theory of semigroups vol 1 and 2, Mathematical Surveys 7 (American Mathematical Society, Providence, RI, 1961 and 1967).

[2] J.M. Howie and M.P.O. Marques-Smith, 'Inverse semigroups generated by nilpotent transformations', Proc. Roy. Soc. Edinburgh Sect. A 99 (1984), 153-162.

[3] L.M. Lima, 'Nilpotent local automorphisms of an independence algebra', Proc Roy. Soc. Edinburgh Sect. A 124 (1994), 423-436.

[4] M.P.O. Marques-Smith and R.P. Sullivan, 'The ideal structure of nilpotent-generated transformation semigroups', Bull. Austral. Math. Soc. 60 (1999), 303-318.

[5] S. Mendes-Gonçalves and R.P. Sullivan, 'Baer-Levi semigroups of linear transformations', Proc. Roy. Soc. Edinburgh Sect. A 134 (2004), 477-499.

[6] S. Mendes-Gonçalves and R.P. Sullivan, 'Maximal inverse subsemigroups of the symmetric inverse semigroup on a finite-dimensional vector space', Comm. Algebra (to appear).

Centro de Matematica

Universidade do Minho

4710 Braga

Portugal
School of Mathematics and Statistics

University of Western Australia

Nedlands WA 6009

Australia 